\title{
Publisher Correction to: Robustly Tuning Bandgaps in Two-Dimensional Soft Phononic Crystals with Criss-Crossed Elliptical Holes
}

\author{
Nan Gao $^{1} \quad$ Yi-lan Huang $^{1} \quad$ Rong-hao Bao $^{1,3 \star} \quad$ Wei-qiu Chen $^{1,2,3,4 \star}$ \\ $\left({ }^{1}\right.$ Department of Engineering Mechanics, Zhejiang University, Hangzhou 310027, China) \\ $\left({ }^{2}\right.$ State Key Lab of CAD \& CG, Zhejiang University, Hangzhou 310058, China) \\ $\left({ }^{3}\right.$ Key Laboratory of Soft Machines and Smart Devices of Zhejiang Province, Zhejiang University, Hangzhou \\ 310027, China) \\ ( ${ }^{4}$ Soft Matter Research Center, Zhejiang University, Hangzhou 310027, China) \\ published online 26 September 2018 \\ (C) The Chinese Society of Theoretical and Applied Mechanics 2018

\section{Publisher Correction to: Acta Mechanica Solida Sinica https://doi.org/10.1007/s10338-018-0044-4}

The original version of this article unfortunately contained mistakes. Due to typesetting errors in Figure 5, figure parts (a) and (b) and parts (d) and (e) were in the wrong order. The same applies to Figure 12, parts (a) and (b).

The original article has been corrected.

\footnotetext{
* Corresponding authors. E-mails:brh@zju.edu.cn; chenwq@zju.edu.cn
} 